Published in final edited form as:

J Neurooncol. 2019 January ; 141(2): 267-276. doi:10.1007/s11060-018-03040-8.

\title{
Magmas inhibition as a potential treatment strategy in malignant glioma
}

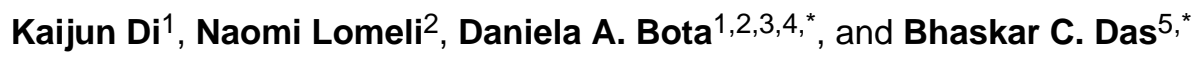 \\ ${ }^{1}$ department of Neurology, University of California Irvine, Irvine, CA, USA; \\ ${ }^{2}$ Department of Pathology \& Laboratory Medicine, University of California Irvine, Irvine, CA, USA; \\ ${ }^{3}$ Department of Neurological Surgery, University of California Irvine, Irvine, CA, USA; \\ ${ }^{4}$ Chao Family Comprehensive Cancer Center, University of California Irvine, Irvine, CA, USA; \\ ${ }^{5}$ Department of Medicine and Pharmacological Sciences, The Icahn School of Medicine at Mount \\ Sinai, NY, USA
}

\section{Abstract}

Purpose-Magmas (mitochondria-associated protein involved in granulocyte-macrophage colony-stimulating factor signal transduction) is a nuclear gene that encodes the mitochondrial import inner membrane translocase subunit Tim16. Magmas is highly conserved, ubiquitously expressed in mammalian cells, and is essential for cell viability. Magmas expression levels are increased in prostate cancers and pituitary adenomas. Moreover, silencing Magmas by RNAi sensitizes pituitary adenoma cells to pro-apoptotic stimuli and induces a G0/G1 accumulation. The aim of this study was to examine whether inhibition of Magmas by small molecule inhibitors could be beneficial for the treatment of malignant gliomas.

Methods-We evaluated the expression of Magmas in patient-derived glioblastoma tissue samples and xenograft models. We studied the feasibility of a small molecule Magmas inhibitor (BT\#9) as a therapeutic agent in stable human glioma cell lines and high-grade patient derived glioma stem-like cells.

Results-Magmas was overexpressed in tissue sections from glioma patients and xenografts. In vivo studies revealed that BT\#9 could cross the blood-brain barrier in the animal model. Magmas inhibition by BT\#9 in glioma cell lines significantly decreased cell proliferation, induced apoptosis along with vacuole formation, and blocked migration and invasion. In addition, BT\#9 treatment decreased the respiratory function of glioma cells, supporting the role that Magmas serves as a ROS (reactive oxygen species) regulator.

Conclusions-This is the first study on the role of Magmas in glioma. Our findings suggest that Magmas plays a key role in glioma cell survival and targeting Magmas by small molecule inhibitors may be a therapeutic strategy in gliomas.

*Co-Corresponding Authors: Daniela A. Bota, MD, PhD, 200 S. Manchester Ave., Suite 206, Orange, CA, 92868, Phone: 714-456-7032; Fax: 714-456-6894, dbota@uci.edu; Bhaskar C. Das, PhD, Department of Medicine and Pharmacological Sciences, The Icahn School of Medicine at Mount Sinai, NY-10029, Phone: 845-300-2153; Fax \#: 212-987-0389, Bhaskar.das@mssm.edu. 


\section{Keywords}

Magmas; glioma; small molecule inhibitor; ROS; Anti-tumor activity

\section{Introduction}

Magmas (mitochondria-associated protein involved in granulocyte-macrophage colonystimulating factor signal transduction) is a nuclear gene identified as a GM-CSF (Granulocyte-macrophage colony stimulating factor) inducible gene in myeloid cells [1]. Magmas encodes the mitochondrial import inner membrane translocase subunit Tim16, and is highly conserved and ubiquitously expressed in all mammalian cells [2, 3]. Increasing evidence suggests a role for Magmas in tumorigenesis. The expression levels of Magmas are barely detectable in normal human prostate, but increase in high-grade carcinoma, independently of mitochondrial content [4]. In addition, Magmas mRNA has been found to be highly expressed in two ACTH-secreting mouse pituitary adenoma cell lines compared to normal mouse pituitary cells, and in 47 out of 64 human pituitary adenomas compared to normal human pituitary cells [5]. Furthermore, decreased expression levels of Magmas in cancer cells are associated with apoptosis. Magmas silencing by RNAi sensitizes pituitary adenoma cells to proapoptotic stimuli and induces a G0/G1 accumulation [5], while overexpression of Magmas increases cell growth and inhibits staurosporine-induced apoptosis as well as DNA fragmentation [6]. Magmas also performs a critical ROS (reactive oxygen species) regulatory function by increasing cell tolerance to oxidative stress and protecting cells from damage and apoptosis [7]. Based on these findings, we hypothesized that inhibition of Magmas by small molecule inhibitors could be beneficial for glioma treatment.

Glioblastoma (GBM) is one of the most aggressive and difficult to treat cancers. Drug delivery across the blood-brain barrier (BBB) and GBM resistance to conventional chemotherapy are two factors that contribute to the poor prognosis of GBM patients. The function of BBB is to efficiently supply the brain with nutrients and oxygen, meanwhile protecting the brain from potentially neurotoxic compounds. The active metabolism of GBM, along with increased expression of Vascular Endothelial Growth Factor (VEGF) and angiogenesis, leads to the abnormal vascularization and a dysfunctional $\mathrm{BBB}$, a major obstacle in brain tumor therapy by preventing the delivery of sufficient quantities of therapeutic agents [8]. Therefore, no matter how potent the drug is, there is no benefit for glioma treatment if it cannot cross the BBB and reach the tumor cells. Accumulating evidence suggests that glioma stem-like cells (GSCs), a small subset of tumor cells situated at the apex of the cellular differentiation hierarchy, are resistant to chemotherapy due to their stem cell-like properties. Although anti-cancer therapies could effectively eliminate the tumor mass, surviving GSCs can re-initiate tumor formation [9]. Thus drugs targeting GSCs may increase therapeutic efficacy and decrease tumor recurrence.

The aim of our study was to investigate the role of Magmas on malignant gliomas, to test the capability of a novel Magmas inhibitor, BT\#9, to cross the BBB, and to evaluate the antitumor function of BT\#9 in human glioma cells and GSCs. 


\section{Materials and Methods}

\section{Chemicals and reagents}

All standard chemicals, buffers and reagents, unless otherwise indicated, were purchased from Sigma Aldrich (St. Louis, MO). BT\#9 was synthesized by Bhaskar C. Das.

\section{Glioma tissues, xenografts and cells}

Institutional Review Board approval was obtained at University of California, Irvine Medical Center. Surgical specimens of brain tumors were obtained from patients who had undergone tumor resection with the neuropathological review completed by a specialized neuropathologist. For GL261 xenografts, five 6-week old BALB/C nu/nu male nude mice were subcutaneously injected with $5 \times 10^{5}$ GL261 cells in 25\% Matrigel (BD Biosciences) into their right and left thighs on both sides. On Day 15 after injection, the mice were sacrificed and tumors were removed and fixed. The established malignant human glioma cell lines, D-54 MG, U-118 MG, U-251 MG and U-87 MG were maintained in DMEM/F-12 medium containing $292 \mu \mathrm{g} / \mathrm{ml}$ glutamine, $1 \%$ penicillin/streptomycin and 10\% FBS (Omega Scientific, Inc. Tarzana, CA). GSC HuTuP01 cells were a gift from Dr. David Panchision [10]. Mesenchymal (Mes) GSCs 1123 Mes and 83 Mes were a gift from Dr. Ichiro Nakano [11]. All GSCs were cultivated in undifferentiated conditions on Fibronectin-coated flasks in 1:1 DMEM:F12 medium (Irvine Scientific, Santa Ana, CA), containing 1\% penicillin/ streptomycin (Gibco/Invitrogen, Grand Island, NY), 10\% BIT9500 (Stem Cell Technologies, Vancouver, BC, Canada), $292 \mu \mathrm{g} / \mathrm{ml} \mathrm{L}$-glutamine (Irvine Scientific), $20 \mathrm{ng} / \mathrm{ml}$ basic Fibroblast Growth Factor (FGF) (PeproTech, Inc., Rocky Hill, NJ), 20 ng/ml Epidermal Growth Factor (EGF) (PeproTech, Inc.), $10 \mu \mathrm{g} / \mathrm{ml}$ Ciprofloxacin (Teva Parenteral Medicines, Inc., Irvine, CA), $10 \mu \mathrm{g} / \mathrm{ml}$ Gentamicin (MP Biomedicals, LLC, Solon, Ohio) and $2.5 \mu \mathrm{g} / \mathrm{ml}$ Amphotericin B (Fisher Scientific, Fair Lawn, NJ). All cells were cultured at $37^{\circ} \mathrm{C}$ in a humidified incubator with $5 \% \mathrm{CO}_{2}$.

\section{Immunohistochemical staining}

VECTASTAIN ABC kit and DAB substrate kit (Vector Laboratories, Inc., Burlingame, CA) were used for immunohistochemical staining. Formalin-fixed, paraffin-embedded tissue sections $(5 \mu \mathrm{m})$ were deparaffinized and rehydrated. After antigen retrieval, the endogenous peroxidase activity was blocked with $1 \% \mathrm{H}_{2} \mathrm{O}_{2}$ in PBS for 20 min. The sections were incubated with 5\% normal goat serum and then exposed to rabbit anti-PAM16 antibody (15321-1-AP, 1:50, ProteinTech Group, Inc., Rosemont, IL) at $4{ }^{\circ} \mathrm{C}$ overnight, followed by incubation with biotinylated anti-rabbit IgG for 1 hour at room temperature. The sections were incubated with $\mathrm{ABC}$ reagent and followed by $\mathrm{DAB}$ substrate solution until desired stain intensity developed. Hematoxylin was used as counterstain. Slides were then mounted with Permount (Fisher Scientific).

\section{Pharmacokinetic (PK) evaluation of BT\#9 in female Balb-C mice}

The $200 \mathrm{mg} / \mathrm{ml}$ Captisol solutions of BT\#9 were used at dosing solutions for animal studies. A dose escalation study was conducted in female Balb-C mice to determine the maximum tolerated dose of this investigational compound. All doses were delivered by IV injection 
into the tail vein. The mice were not fasted and water was available ad libitum. After examining several IV doses ranging from $0.1 \mathrm{mg} / \mathrm{kg}$ to $50 \mathrm{mg} / \mathrm{kg}$ BT\#9, a dose of $30 \mathrm{mg} / \mathrm{kg}$ which was well tolerated by the animals with very little observed negative side-effects, was selected for the pilot PK study. Ten time points ( 3 mice per time point) were selected for the pilot PK study. Plasma was collected at each time point, as well as perfused brains to assess blood brain barrier permeation of BT\#9.

Blood samples were taken from anesthetized mice by cardiac puncture with a needle and syringe at approximately time $0,5,10,20,30,60,120,240,480$ and 720 minutes. After each blood sample was taken, all mice were euthanized via isoflurane anesthesia and exsanguination, with death ensured by thoracotomy. Each animal was then perfused with saline and the brain was harvested and frozen for tissue levels of BT\#9. The plasma and brain BT\#9 concentrations were determined using validated LC/MS/MS bioanalytical methods.

After the plasma LC/MS/MS analysis was completed, data was analyzed using WinNonLin Pharmacokinetic Software, version 3.1 (Pharsight, Mountain View, CA). For the brain tissue, concentration data (normalized to tissue weight, expressed as ng/g BT\#9) was plotted as a mean concentration-time profile to show drug elimination from brain tissue.

\section{3-[4,5-Dimethylthiazol-2-yl]-2,5-diphenyltetrazolium bromide (MTT) assay}

The cells were seeded at approximately $1 \times 10^{4} /$ well in a final volume of $200 \mu \mathrm{l}$ in 96-well microtiter plates. Before testing, MTT solution ( $5 \mathrm{mg} / \mathrm{ml} ; 20 \mu \mathrm{l} / \mathrm{well})$ was added and cells were incubated at $37^{\circ} \mathrm{C}$ for 5 hours. The culture medium was then aspirated and DMSO ( $200 \mu \mathrm{l} /$ well, Fisher Scientific) was added to dissolve the dark blue crystals. The absorbance was measured at a wavelength of $570 \mathrm{~nm}$.

\section{Western blotting}

Antibodies used were Cleaved Caspase-3 (Asp175) (\#9661, Cell Signaling Technology, Inc., Danvers, MA) $\beta$-actin (NB600-501, Novus Biological LLC, Littleton, CO). The images were exposed by G:BOX from Syngene.

\section{Apoptosis assay}

$\mathrm{U}-251$ cells were treated with $10 \mu \mathrm{M}$ of BT\#9 for 24 hours, and apoptotic cells were measured using the Annexin V: FITC Apoptosis Detection kit (BD Biosciences, Franklin Lakes, NJ). Samples were then analyzed by flow cytometry using BD Bioflow.

\section{Wound closure assay}

Cells were seeded in a 24 -well plate at $1 \times 10^{5}$ cells/well and were allowed to grow overnight. The monolayer was then scratched with a pipette tip, washed with PBS twice, and treated with Magmas inhibitor. At the end of each incubation, cell migration into the scratched area was photographed. 


\section{Invasion assay}

Invasion assay was performed using BD BioCoat ${ }^{\mathrm{TM}}$ Matrigel ${ }^{\mathrm{TM}}$ Invasion Chamber with $8 \mu \mathrm{m}$ PET membrane (BD Biosciences). Cells were seeded in medium without serum, and medium containing $1.5 \%$ FBS was used as chemoattractant. After an incubation of 24 hours at $37^{\circ} \mathrm{C}$, non-invasive cells were removed and invading cells were fixed with $100 \%$ methanol. Cells were then stained in Hematoxylin.

\section{Seahorse XF24 metabolic flux analysis}

Mitochondrial respiratory function was assessed using the Seahorse XF24 Extracellular Flux Analyzer. Oxygen consumption rates (OCR, pmol/min) and extracellular acidification rates (ECAR, $\mathrm{mpH} / \mathrm{min}$ ) were measured using the Cell Mito Stress Kit (Seahorse Bioscience, Billerica, MA). The cells, D-54 MG $\left(8.0 \times 10^{4} /\right.$ well $)$ and U-251 MG $\left(1.0 \times 10^{5} /\right.$ well $)$ were plated on Seahorse XF24 cell culture microplates. Baseline rates were measured at $37^{\circ} \mathrm{C}$ three times before the sequential injection of the mitochondrial inhibitiors: oligomycin (2 $\mu \mathrm{M}), \mathrm{FCCP}(0.5 \mu \mathrm{M})$ and lastly roteneone $(1 \mu \mathrm{M})$ and antimycin A $(1 \mu \mathrm{M})$ in D54-MG. The inhibitor concentrations used for the U-251 MG cell line were: oligomycin $(2 \mu \mathrm{M})$, FCCP (1 $\mu \mathrm{M})$, and roteneone $(1 \mu \mathrm{M})$ plus antimycin $\mathrm{A}(1 \mu \mathrm{M})$. Three OCR and ECAR measurements were taken after addition of each inhibitor. All measurement was normalized to protein content per well. OCR and ECAR data was collected using the XF4 Wave software (Seahorse Bioscience). Basal OCR levels were analyzed using the Agilent Seahorse XF Cell Mito Stress Test Report Generator. Basal OCR respiration parameter $=$ (last rate measurement before Oligomycin injection) - (Non-mitochondrial respiration rate). Nonmitochondrial oxygen consumption $=$ minimum rate measurement after Rotenone/ Antimycin A injection. Basal ECAR levels were calculated as the average of the first three measurements before Oligomycin injection. Data was graphed and analyzed using GraphPad Prism 5 (GraphPad Software, La Jolla, CA). Statistical significance was measured by student's paired t-test.

\section{Statistical Analysis}

Statistical analyses were performed and graphs generated using Prism 5 (GraphPad). All values were presented as mean \pm standard error of the mean (S.E.M.) when at least 3 observations were available. Statistical significance was measured by unpaired $t$-tests or oneway ANOVA unless otherwise indicated.

\section{Results}

\section{Magmas over-expression is observed in glioblastoma tumor tissues}

Magmas expression in several types of human tumors has been shown to be significantly higher than the levels present in normal tissues. However, very little is known regarding the role of Magmas in gliomas. We first evaluated the expression of Magmas in gliomas using immunohistochemical analysis (Fig. 1a). High Magmas expression was detected in patientderived GBM tissues as well as tumor sections from nude mice subcutaneously injected with GL261 xenograft, but much lower and/or undetectable expression was noted in normal 
brains (representative results shown). These results suggest that targeting Magmas may be a potential therapeutic strategy in gliomas.

\section{Magmas inhibitor may cross the blood-brain barrier and enters brain as a potential target organ}

A highly conserved region important for Magmas activity was identified by sequence homology and functional mutagenesis. Using structural data and molecular modeling, several compounds designed to bind to Magmas were synthesized. Among them, the most active compound (BT\#9) was studied for functional interactions with Magmas [12] and used in our study (Fig. 1b). First, we evaluated the pharmacokinetics (PK) and metabolism of BT\#9 in vivo using female Balb-C mice. An intravenous dose of BT\#9 $(30 \mathrm{mg} / \mathrm{kg})$ was chosen for the pilot PK study, and plasma was collected at ten time points $(0,5,10,20,30$, 60, 120, 240, 480 and 720 minutes) for the pilot PK study. Meanwhile, perfused brains were also collected to assess blood brain barrier permeation of BT\#9. After an intravenous dose of BT\#9 (30 mg/kg), the maximum plasma concentration could be seen at 5 minutes, with a Cmax of $4497.06 \mathrm{ng} / \mathrm{mL}$. The apparent half-life of BT\#9 after IV dosing was 209.2 minutes (Table 1). By comparing the plasma concentration-time profile of BT\#9 (gray line) to the levels of BT\#9 in the brain (black line), we found that while the plasma level of BT\#9 reached a Cmax within 5 minutes and obviously eliminated by 720 minutes, brain levels of BT\#9 increased over the first 240 minutes after IV exposure and then slowly decrease (Fig. 1c). It is possible that BT\#9 is sequestered in the lipid rich environment of the brain and leeches out over time. It is also possible that BT\#9 binds to a specific receptor site in the brain and is not eliminated quickly as it is in the plasma. Since measurable BT\#9 levels are seen in the brain, as well as the plasma (and other tissues, data not shown), it is indicative that BT\#9 is getting to the target tissue (brain) and remaining there, where it can exert its pharmacological actions.

\section{Magmas inhibitor has a dose- and time-dependent cytotoxic effect on glioma cells}

Next, we focused on the effect of BT\#9 on glioma cells. In order to determine if BT\#9 is toxic to glioma cells, several established malignant human glioma cell lines (D-54 MG, U-118 MG, U-251 MG and U-87 MG) were chosen to perform an MTT assay to evaluate the effect of BT\#9 on cell proliferation. As shown in Fig. 2a and 2b, BT\#9 significantly inhibited the growth of those glioma cell lines in a dose- and time-dependent manner, suggesting that $\mathrm{BT} \# 9$ exerts cytotoxic effects on glioma cells.

GSCs are known to contribute to tumorigenesis and radiation resistance in malignant glioma [13], therefore targeting GSCs is very important in glioma therapy. We tested the response to BT\#9 among several GSCs derived from high-grade glioma patients. As shown in Fig. 2c, BT\#9 significantly inhibited the proliferation in all cell types tested. The similar sensitivity of high-grade GSCs and the stable glioma cell lines to BT\#9 suggests a potential therapeutic role of BT\#9 in gliomas.

\section{Magmas inhibitor induces apoptosis, inhibits cell migration, and invasion in glioma cells}

The growth inhibition induced by BT\#9 was accompanied with apoptosis induction. BT\#9 treatment led to a significant up-regulation of cleaved caspase-3 (Fig. 3a), an early step in 
the apoptosis cascade leading to nuclear fragmentation. Induction of apoptosis by BT\#9 was confirmed by flow cytometry (Fig. 3b). Meanwhile, cells treated with BT\#9 for 24 hours revealed vacuole formation in a dose-dependent manner (Fig. 3c). Vacuole formation in mammalian cells is a well-known morphological phenomenon when cells are exposed to kinds of pathogens and compounds, and always accompanies cell death [14].

Since the invasive behavior of malignant gliomas limits the effectiveness of local therapies and contributes to their poor prognosis [15], wound closure assay (Fig. 4a) was performed to compare the migrative capability between cells with or without BT\#9 treatment. The results revealed that while control cells were able to migrate in and almost fill up the gap within 24 hours, the cells treated with $10 \mu \mathrm{M}$ of BT\#9 stopped migrating. The role of BT\#9 on cell invasion was further investigated and confirmed by Matrigel invasion assay (Fig. 4b).

\section{Magmas inhibition in malignant glioma cell lines alters mitochondrial respiration function}

In addition to its roles as an inner mitochondrial membrane-associated protein and a component of mitochondrial protein translocation machinery, Magmas also plays a role in regulating the activity of mitochondrial electron transport chain (ETC) complexes [7]. To investigate the effect of Magmas inhibition on the cellular bioenergetics of glioma cell lines D-54 MG and U-251 MG, we assessed basal cellular oxygen consumption rate (OCR) and basal extracellular acidification rate (ECAR) using the Seahorse Biosciences Extracellular Flux Analyzer. OCR is a measure of oxidative phosphorylation (OXPHOS), whereas ECAR is an indicator of glycolysis. Magmas inhibition using BT\#9 decreased basal OCR levels in a dose-dependent manner in the D-54 MG (Fig. 5a, b) and U-251 MG (Fig. 5c, d) lines after 48 hours treatment. In addition, $10 \mu \mathrm{M}$ of BT\#9 significantly reduced basal ECAR levels in the D54-MG line (Fig. 5e, f) and a modest decrease in the U-251 MG line (Fig. 5g, h).

\section{Discussion}

Magmas was originally identified as protein involved in GM-CSF signaling in mammalian cells [1] and was later found to have a role in mitochondrial protein transport in yeast [16, 17]. The expression pattern of Magmas in human disease has been reported in human prostate [4] and pituitary adenomas [5], but little is known in glioma. In this study, we showed that Magmas is overexpressed in tissue sections from glioma patients and xenografts (Fig. 1a), leading us to explore the feasibility of targeting Magmas by small molecule inhibitors as a potential therapeutic strategy in gliomas. Magmas inhibitor BT\#9 (Fig. 1b) directly binds to Magmas, and has been identified as the most active compound among several small molecule Magmas inhibitors [12]. Our in vivo study demonstrated that administration of BT\#9 in Balb-c mice resulted in elevated levels of BT\#9 in the brain over the first 240 minutes after IV exposure and slowly decreased afterwards (Fig. 1c), indicating that BT\#9 could cross the blood-brain barrier and enter the brain, where it can exert its pharmacological actions.

The in vitro anti-tumor effect of Magmas was performed using glioma cells. Magmas inhibition by BT\#9 had significant cytotoxic effect and repressed growth in a dose- and time-dependent manner in glioma cell lines as well as GSCs (Fig. 2). The growth inhibition induced by BT\#9 was accompanied with apoptosis induction as indicated by a significant 
up-regulation of cleaved caspase-3, as well as vacuole formation (Fig. 3). This result is consistent with the previous studies on the effect of Magmas on cell apoptosis [5, 6]. The invasive behavior of malignant gliomas contributes to their poor prognosis [15], and our studies indicated that BT\#9 could inhibit the migration and invasion capability of glioma cells (Fig. 4).

Cancer cells must survive and adapt to challenging microenvironments, specifically in conditions where tumor growth makes oxygen and glucose scarce [18]. Therefore, mitochondrial changes represent a significant part of cancer cell biology. These restrictive conditions require fundamental changes in cellular metabolism, including the respiratory transition from oxidative phosphorylation to glycolysis, which is known as the Warburg effect [19]. Although adaptation and survival in hypoxic conditions are hallmarks of aggressive tumors [20], little is known about the mitochondrial mechanisms involved in the hypoxic adaptation. Developing tumors inevitably deplete their oxygen and nutrient supplies as they proliferate, only tumor cells which adapt to hypoxia survive and become more aggressive/invasive [20]. Glioblastoma is a tumor characterized by extensive hypoxiainduced phenotypic changes such as abnormal vascular proliferation and necrosis [21, 22]. Previous studies have shown that hypoxic adaptation is a prerequisite for glial tumor progression and that the degree of hypoxia correlates with tumor grade, clinical aggressiveness, and increased risk of recurrence after surgery in patients [23]. In this study, our data showed that Magmas inhibition by BT\#9 resulted in impaired respiratory function as indicated by decreased OCR and ECAR in glioma cells (Fig. 5). These results suggest that Magmas overexpression in glioma may enable the mitochondria to function more efficiently in response to high metabolic demand and hypoxic conditions.

Taken together, our findings demonstrate that Magmas is overexpressed in tissue sections from glioma patients and xenografts. In vivo studies showed that a novel Magmas small molecule inhibitor, BT\#9, could cross the blood-brain barrier in mice. In vitro studies revealed that BT\#9 significantly decreased cell proliferation, induced apoptosis, and blocked migration and invasion. In addition, BT\#9 treatment resulted in a decrease in respiratory function of glioma cells. These findings suggest that Magmas may serve as a potential therapeutic target in glioma, and its inhibition might prove to be a valuable strategy for future cancer treatments in hypoxic conditions, which are resistant to traditional treatments.

\section{Acknowledgements}

This study was supported by donations from grateful patients to Dr. Bota laboratory and the UCI Cancer Center Award Number P30CA062203 from the National Cancer Institute (NCI/NIH). Dr. Das is thankful to Dr. Michael Baltezor (Director, Biotechnology Innovation and Optimization Center, Kansas City, KS) for the PK studies.

\section{References}

1. Jubinsky PT, Messer A, Bender J, Morris RE, Ciraolo GM, Witte DP et al. (2001) Identification and characterization of Magmas, a novel mitochondria-associated protein involved in granulocytemacrophage colony-stimulating factor signal transduction. Exp Hematol 29:1392-1402 [PubMed: 11750097]

2. Jubinsky PT, Short MK, Mutema G, Witte DP (2003) Developmental expression of Magmas in murine tissues and its co-expression with the GM-CSF receptor. J Histochem Cytochem 51:585596 [PubMed: 12704206] 
3. Peng J, Huang CH, Short MK, Jubinsky PT (2005) Magmas gene structure and evolution. In Silico Biol 5:251-263 [PubMed: 15984936]

4. Jubinsky PT, Short MK, Mutema G, Morris RE, Ciraolo GM, Li M (2005) Magmas expression in neoplastic human prostate. J Mol Histol 36:69-75 [PubMed: 15704001]

5. Tagliati F, Gentilin E, Buratto M, Mole D, degli Uberti EC, Zatelli MC (2010) Magmas, a gene newly identified as overexpressed in human and mouse ACTH-secreting pituitary adenomas, protects pituitary cells from apoptotic stimuli. Endocrinology 151:4635-4642 [PubMed: 20719856]

6. Tagliati F, Gagliano T, Gentilin E, Minoia M, Mole D, Delgi Uberti EC et al. (2013) Magmas overexpression inhibits staurosporine induced apoptosis in rat pituitary adenoma cell lines. PLoS One 8:e75194 [PubMed: 24069394]

7. Srivastava S, Sinha D, Saha PP, Marthala H, D’Silva P (2014) Magmas functions as a ROS regulator and provides cytoprotection against oxidative stress-mediated damages. Cell Death Dis 5:e1394 [PubMed: 25165880]

8. van Tellingen O, Yetkin-Arik B, de Gooijer MC, Wesseling P, Wurdinger T, de Vries HE (2015) Overcoming the blood-brain tumor barrier for effective glioblastoma treatment. Drug Resist Updat 19:1-12 [PubMed: 25791797]

9. Kim SS, Harford JB, Pirollo KF, Chang EH (2015) Effective treatment of glioblastoma requires crossing the blood-brain barrier and targeting tumors including cancer stem cells: The promise of nanomedicine. Biochem Biophys Res Commun 468:485-489 [PubMed: 26116770]

10. Pistollato F, Chen HL, Rood BR, Zhang HZ, D'Avella D, Denaro L et al. (2009) Hypoxia and HIF1alpha repress the differentiative effects of BMPs in high-grade glioma. Stem Cells 27:7-17 [PubMed: 18832593]

11. Mao P, Joshi K, Li J, Kim SH, Li P, Santana-Santos L et al. (2013) Mesenchymal glioma stem cells are maintained by activated glycolytic metabolism involving aldehyde dehydrogenase $1 \mathrm{~A} 3$. Proc Natl Acad Sci U S A 110:8644-8649 [PubMed: 23650391]

12. Jubinsky PT, Short MK, Ghanem M, Das BC (2011) Design, synthesis, and biological activity of novel Magmas inhibitors. Bioorg Med Chem Lett 21:3479-3482 [PubMed: 21514823]

13. Bao S, Wu Q, McLendon RE, Hao Y, Shi Q, Hjelmeland AB et al. (2006) Glioma stem cells promote radioresistance by preferential activation of the DNA damage response. Nature 444:756760 [PubMed: 17051156]

14. Shubin AV, Demidyuk IV, Komissarov AA, Rafieva LM, Kostrov SV (2016) Cytoplasmic vacuolization in cell death and survival. Oncotarget 7:55863-55889 [PubMed: 27331412]

15. Lim DA, Cha S, Mayo MC, Chen MH, Keles E, VandenBerg S et al. (2007) Relationship of glioblastoma multiforme to neural stem cell regions predicts invasive and multifocal tumor phenotype. Neuro Oncol 9:424-429 [PubMed: 17622647]

16. Frazier AE, Dudek J, Guiard B, Voos W, Li Y, Lind M et al. (2004) Pam16 has an essential role in the mitochondrial protein import motor. Nat Struct Mol Biol 11:226-233 [PubMed: 14981507]

17. Kozany C, Mokranjac D, Sichting M, Neupert W, Hell K (2004) The J domain-related cochaperone Tim16 is a constituent of the mitochondrial TIM23 preprotein translocase. Nat Struct Mol Biol 11:234-241 [PubMed: 14981506]

18. Kato K, Ogura T, Kishimoto A, Minegishi Y, Nakajima N, Miyazaki M et al. (2002) Critical roles of AMP-activated protein kinase in constitutive tolerance of cancer cells to nutrient deprivation and tumor formation. Oncogene 21:6082-6090 [PubMed: 12203120]

19. Bayley JP, Devilee P (2010) Warburg tumours and the mechanisms of mitochondrial tumour suppressor genes. Barking up the right tree? Curr Opin Genet Dev 20:324-329 [PubMed: 20304625]

20. Gillies RJ, Gatenby RA (2007) Adaptive landscapes and emergent phenotypes: why do cancers have high glycolysis? J Bioenerg Biomembr 39:251-257 [PubMed: 17624581]

21. Brat DJ, Castellano-Sanchez A, Kaur B, Van Meir EG (2002) Genetic and biologic progression in astrocytomas and their relation to angiogenic dysregulation. Adv Anat Pathol 9:24-36 [PubMed: 11756757]

22. Brat DJ, Castellano-Sanchez AA, Hunter SB, Pecot M, Cohen C, Hammond EH et al. (2004) Pseudopalisades in glioblastoma are hypoxic, express extracellular matrix proteases, and are formed by an actively migrating cell population. Cancer Res 64:920-927 [PubMed: 14871821] 
23. Evans SM, Judy KD, Dunphy I, Jenkins WT, Hwang WT, Nelson PT et al. (2004) Hypoxia is important in the biology and aggression of human glial brain tumors. Clin Cancer Res 10:81778184 [PubMed: 15623592] 
A



B

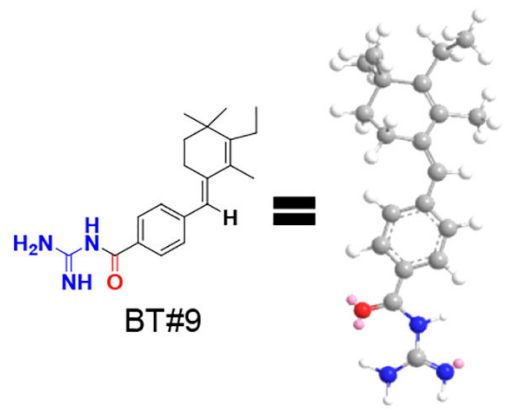

C

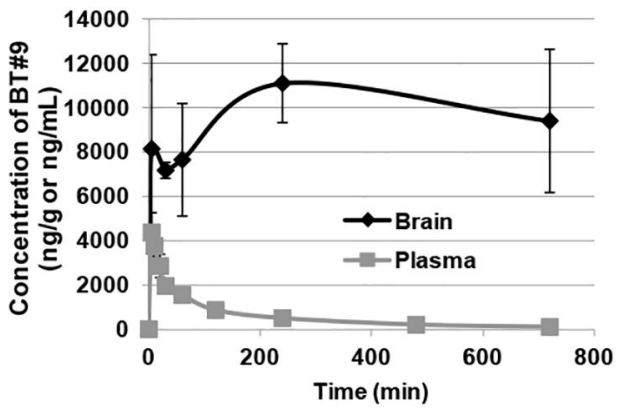

Fig. 1.

a Representative immunostaining of Magmas in human normal brain tissue, human GBM patient tissue, and mouse GL261 xenografts which were subcutaneously injected into nude mice. Magmas staining was performed using paraffin-embedded sections from tissue mass. Microscopic magnification was x200 (upper) and x400 (lower). b Chemical structure of small molecule Magmas inhibitor BT\#9. c Concentration-time profile of BT\#9 in brain homogenate (ng/g) vs plasma (ng/mL). 
A
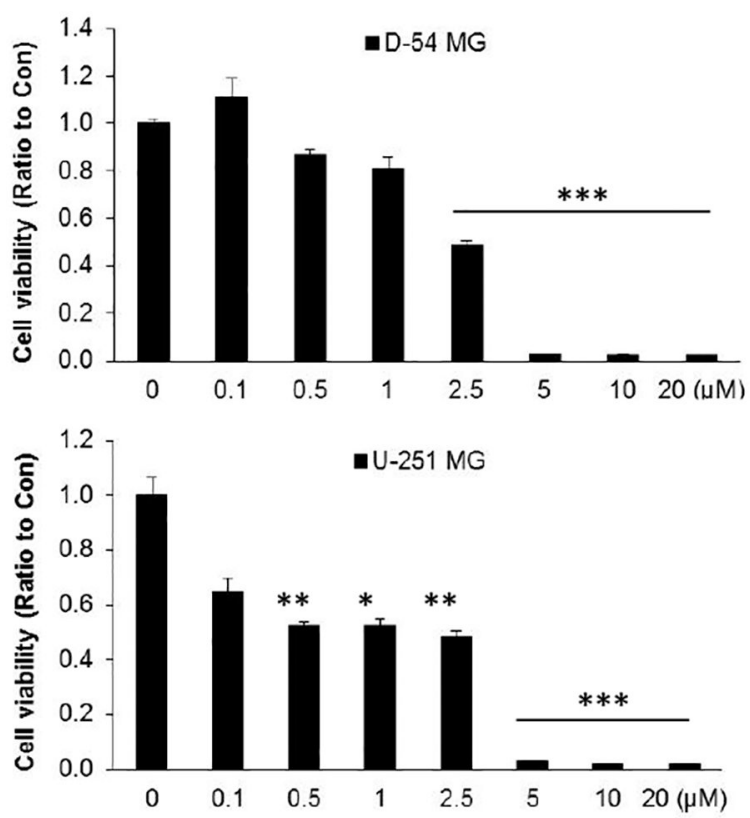

B
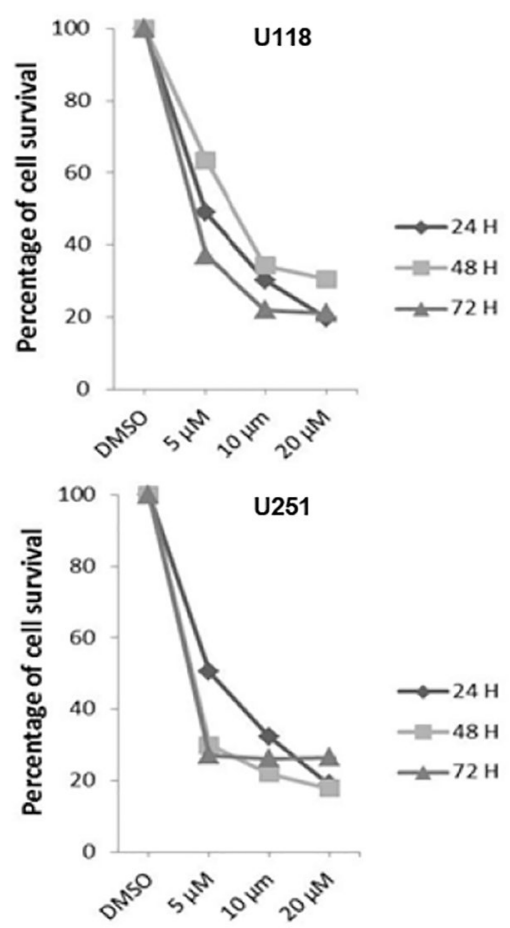
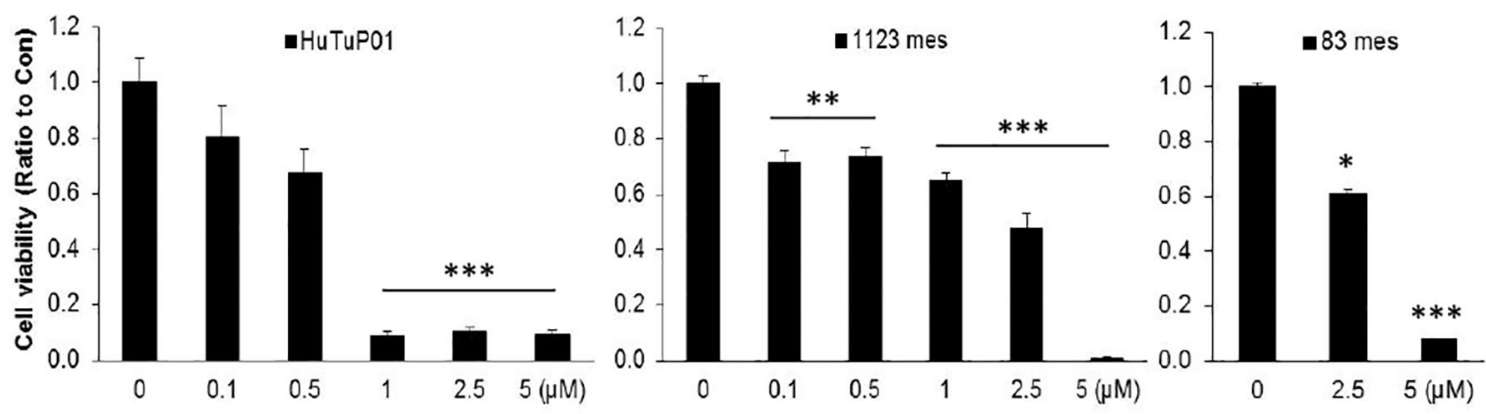

Fig. 2.

Magmas inhibition by BT\#9 has a dose- and time-dependent cytotoxic effect on glioma cells. Established malignant human glioma cell lines, D-54 MG and U-251 MG (a) and human GSCs HuTuP01, 1123 Mes and 83 Mes (c) were incubated for 3 days with increasing concentrations of BT\#9. b U-118 MG and U-251 MG cells were treated with $5 \mu \mathrm{M}, 10 \mu \mathrm{M}$ or $20 \mu \mathrm{M}$ of BT\#9 for 24,48 or 72 hours. Cell viability was measured by MTT assay. The relative numbers of proliferating cells compared with control are presented as the mean \pm SEM. $* p<0.05, * * p<0.01, * * * p<0.001$. 
A
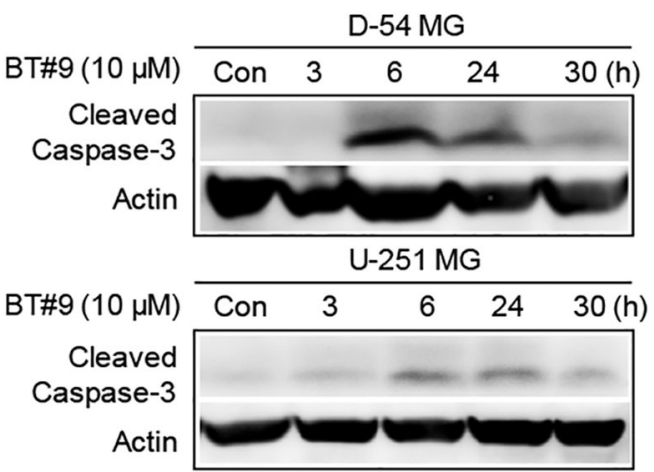

C

U118
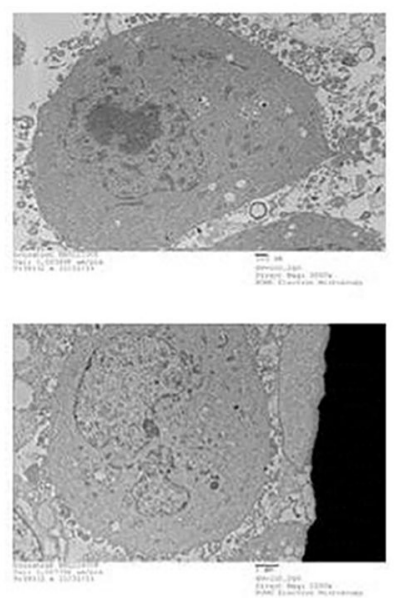

U251

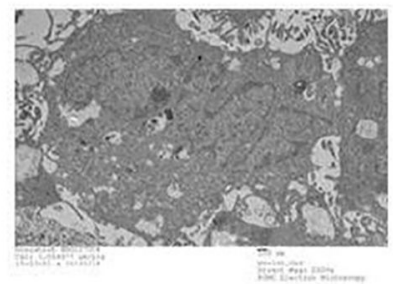

M.I 5uM
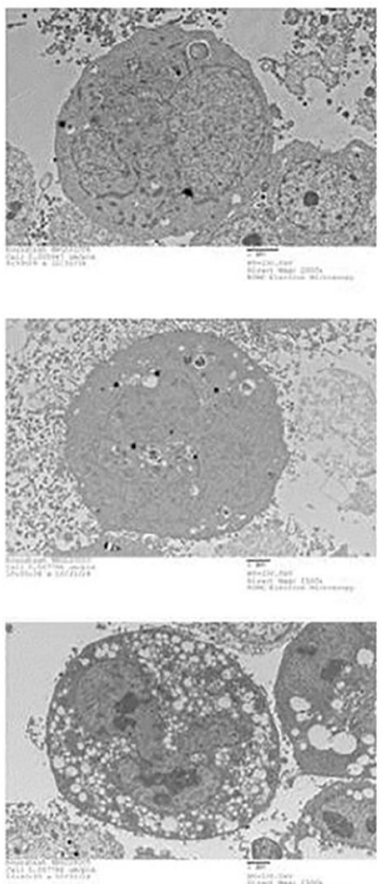

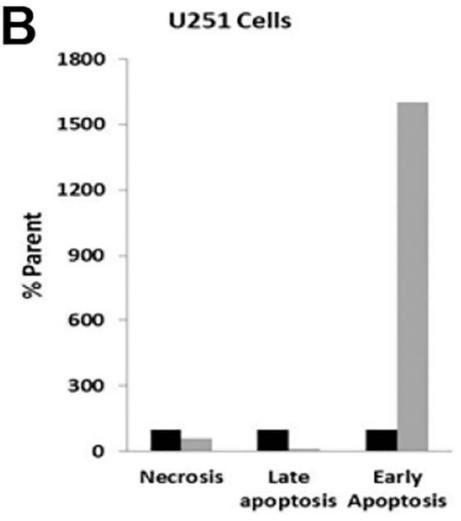

M.I 10uM
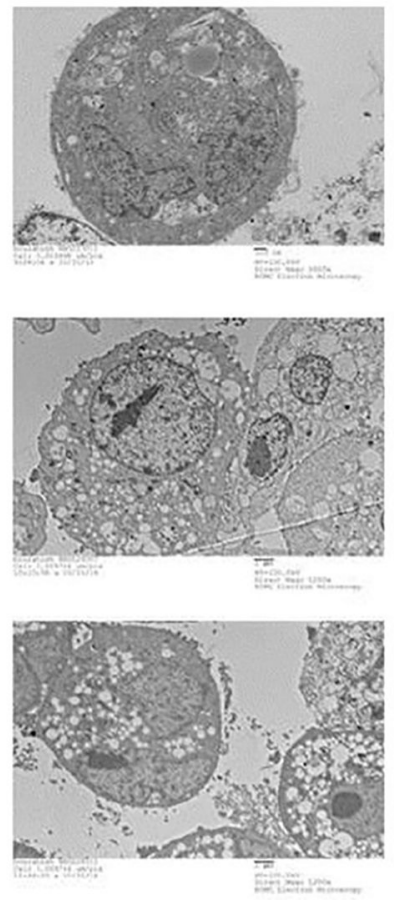

Fig. 3.

Magmas inhibitor BT\#9 induces apoptosis and vacuole formation in glioma cells. a D-54 and $\mathrm{U}-251$ cells were treated with $10 \mu \mathrm{M}$ of $\mathrm{BT} \# 9$ for indicated time points. Western blot was used to detect cleaved caspase-3. Actin was the internal control. b U-251 cells were treated with $10 \mu \mathrm{M}$ of BT\#9 for 24 hours, and cell cycle was analyzed by flow cytometry. c The cells treated with BT\#9 $(10 \mu \mathrm{M})$ for 24 hours were harvested and fixed with formaldehyde and given to microscopy facility for electron microscopy processing and imaging. 
A

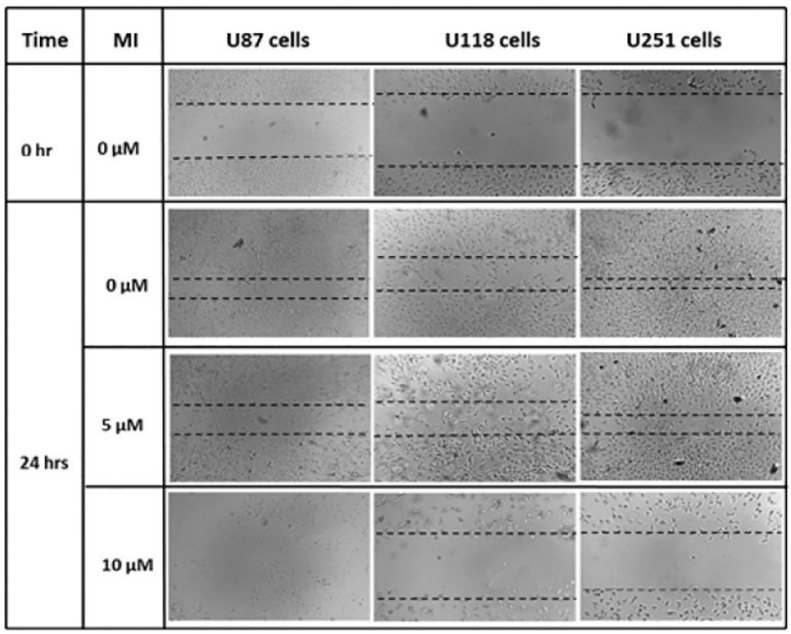

B

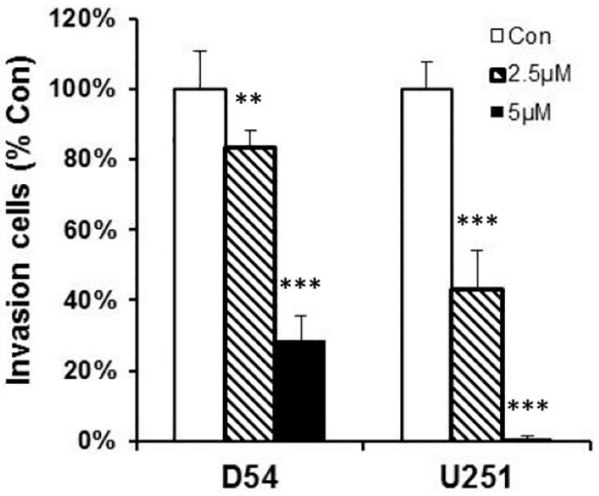

Fig. 4.

Magmas inhibitor BT\#9 inhibits cell migration and invasion in glioma cells. a Wound closure assay was performed using U-87, U-118 and U-251 cells with or without of BT\#9 (5 $\mu \mathrm{M}$ and $10 \mu \mathrm{M})$ treatment for 24 hours. At the end of incubation, the cells migrated into the scratched area was photographed, $\mathbf{b}$ Invasion capability of cells treated or untreated with BT\#9 $(2.5 \mu \mathrm{M}$ and $5 \mu \mathrm{M})$ for 24 hours was analyzed using matrigel invasion chambers. The average of invaded cells was normalized to un-treated control. $* * p<0.01, * * * p<0.001$. 
A

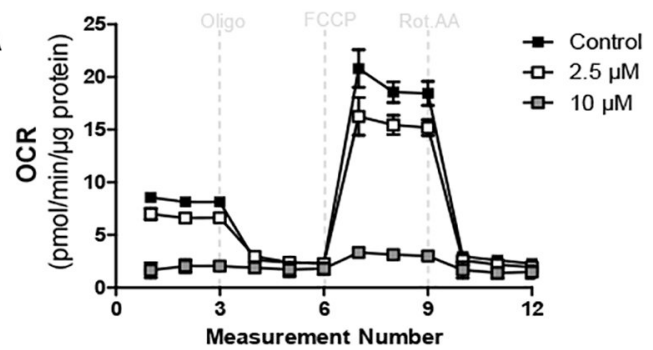

C

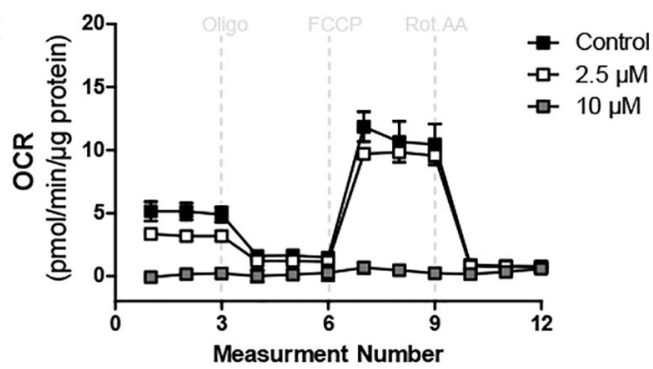

E

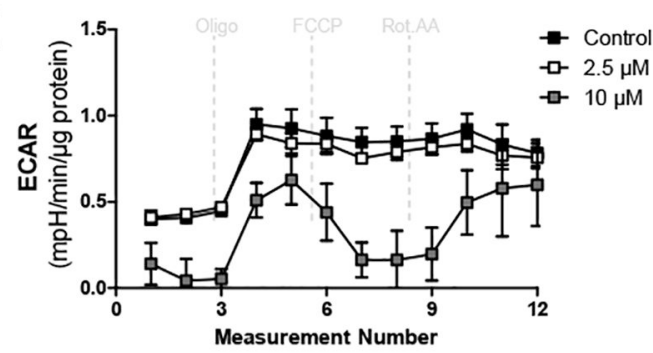

G

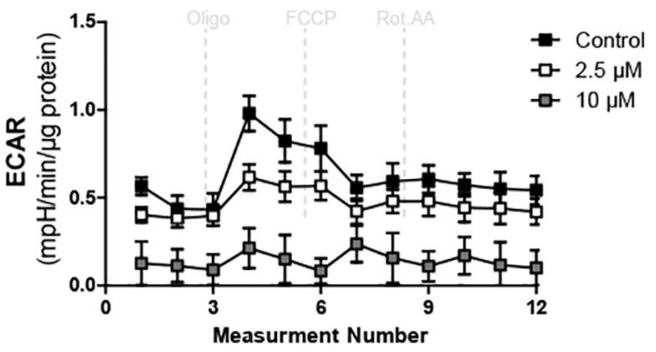

B

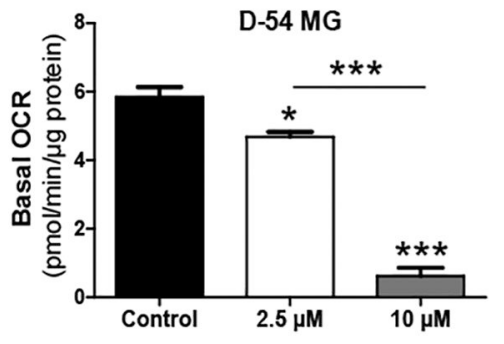

D

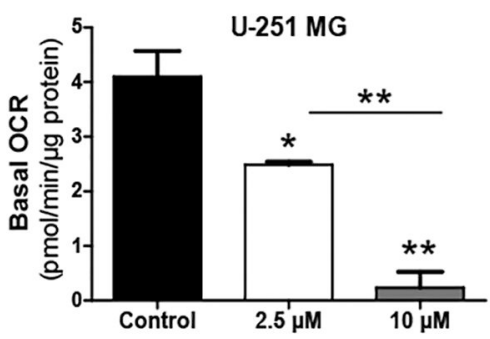

$\mathbf{F}$

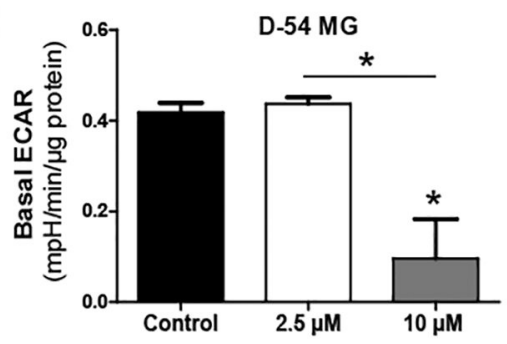

H

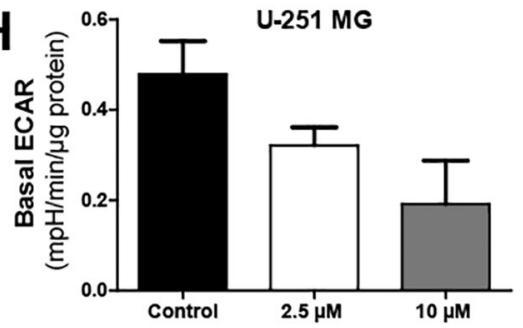

Fig. 5.

Magmas inhibition impaired mitochondrial respiration in glioma cells. Mitochondrial OCR profile of (a) D-54 MG and (c) U-251 MG treated with $2.5 \mu \mathrm{M}$ and $10 \mu \mathrm{M} \mathrm{BT \# 9} \mathrm{for} 48$ hours. Quantitative analysis of basal OCR levels in (b) D-54 MG and (d) U-251 MG indicates a dose-dependent decrease in oxygen consumption. ECAR profile of (e) D54-MG and (g) U-251 MG treated with $2.5 \mu \mathrm{M}$ and $10 \mu \mathrm{M} \mathrm{BT \# 9} \mathrm{for} 48$ hours, (f) $10 \mu \mathrm{M} \mathrm{BT \# 9}$ significantly reduced basal ECAR levels in the D54-MG lines and (h) a mild decrease in the $\mathrm{U}-251 \mathrm{MG}$ line. Data graphed as mean $\pm \mathrm{SEM}, \mathrm{n}=3$ per treatment group. $* P<0.05$, $* * P<0.01, * * * P<0.001$ 
Table 1.

Pharmacokinetic Parameters of Intravenously Dosed BT\#9

\begin{tabular}{|l|l|l|}
\hline Parameter & Unit & Plasma BT\#9 \\
\hline $\mathbf{n}$ & & 3 \\
\hline Dose & $\mathbf{~ m g} / \mathbf{k g}$ & 30 \\
\hline Slope & $\mathbf{1 / m i n}$ & $0.003733 \pm 0.001364$ \\
\hline Half life & $\mathbf{m i n}$ & $209.2 \pm 96.7$ \\
\hline Tmax & $\mathbf{m i n}$ & $5.0 \pm 0.00$ \\
\hline Cmax & $\mathbf{n g} / \mathbf{m L}$ & $4497.06 \pm 770.10$ \\
\hline AUC $(\mathbf{0}-\infty)$ & $\mathbf{m i n}$ ) & $464321.57 \pm 14168.00$ \\
\hline Volume of Distribution & $\mathbf{m L} / \mathbf{k g}$ & $19602.07 \pm 9022.15$ \\
\hline Clearance & $\mathbf{m L} / \mathbf{m i n} / \mathbf{k g}$ & $64.97 \pm 2.35$ \\
\hline
\end{tabular}

